\title{
Developing the Human Factor in an Integrated Health Organization
}

\section{Maria Luz Jauregui García ${ }^{1 *}$, Maria Luisa Merino Hernández ${ }^{1}$, Arantxa Urruzola Lizarribar ${ }^{1}$, Nagore Lora Laca ${ }^{1}$, Iobat Jiménez Jauregui ${ }^{2}$ and Francisco Serna Rodríguez ${ }^{3}$}

${ }^{1}$ OSI Tolosaldea Osakidetza, Spain

${ }^{2}$ Master's Student Health Management, University of Deusto, Spain

${ }^{3}$ Central Osakidetza Organization, Spain

*Corresponding Author: Maria Luz Jauregui García, OSI Tolosaldea Osakidetza, Spain.

DOI: 10.31080/ASNH.2020.04.0732
Received: June 24, 2020

Published: July 16, 2020

(C) All rights are reserved by Maria Luz

Jauregui García., et al.

\section{Abstract}

This article describes a formative and participatory understanding with the aim of fostering a relational culture that facilitates the transition to a patient-centered care model by improving communication. It consists in transmitting the advances of neuroscience and technology related to unit professionals through a common communication code that highlights the importance of the human factor. In addition, the values of the organization are worked, based on professionals, through collaborative work in each UAP.

It analyses ns results of the intervention, highlighting do that professionals manifest as very positive the being able to share values in the way of understanding human relationships. They also show that the project has allowed us to generate discussion and reflection on how to communicate.

Working these values from the perspective of professionals acts as a facilitator to feel the culture of the organization as their own.

Participants can improve their personal relationship as this intervention encourages the sharing of beliefs and willgo in a relaxed environment.

Keywords: UAP; Fostering; OSI

\section{Abbreviations}

UAP: Primary Care Unit; OSI: Integrated Health Organization; AAC: Customer Service Area

\section{Introduction}

According to various studies, effective and participatory communication as well as confidence in the doctor-patient relationship has a great influence on health outcomes, in satisfaction with the health system and is key to a more adequate use of health resources.

There is a consensus in the scientific world that believes that effective communication, within a context of education and respect, predicts greater well-being and better health. In addition, there is evidence that effective communication improves outcomes and clinical diagnosis, adherence to treatment and emotional well-being of the patient and decreases the demand for consultations [1].

It has also been observed that with good communication there is an increase in therapeutic compliance and decreases anxiety, pain and the onset of other associated problems [2].

Non-participatory communication increases communication problems and negatively affects the relationship with patients and their health status. $54 \%$ of complaints and $45 \%$ of patient concerns are not collected. Doctors often take 18" to interrupt the patient when they start recounting their concerns and won't let them talk [3]. 
It has also been seen that non-participatory communication increases the expenditure and unnecessary use of health services [2]

It has been established that good communication within the framework of the patient-patient relationship generates In this relationship improves satisfaction, involves the patient in decisionmaking, leads to more realistic patient expectations, produces a more effective practice [4-6] and is associated with positive health outcomes, including emotional health and help in symptom resolution [7].

Several studies [8-10] have analyzed the influence of the doctor-patient relationship on osteomuscular pain pictures. Patients receiving a patient-centered approach by physicians trained in communication skills versus regular care for patients with chronic skeletal musculoskeletal pain and fibromyalgia have been observed to show an improvement in pain perception and anxiety. It seems that even the effect of the doctor-patient relationship at 6 months after rehabilitation in patients with chronic lower back pain is clearer than at the end of rehabilitation.

A systematic review [11] of randomized intervention trials conducted in primary care with the aim of altering the interaction between patients and physicians to assess the effect of interventions on improving patients' health and well-being concluded that the positive effects on health outcomes reached statistical significance in nearly half of the studies. However, most had significant methodological problems, which could have affected the findings of the review.

In this sense, a recent Cochrane review of 43 randomized trials, most conducted in the context of primary care, revealed that the training of professionals to improve their ability to share control with patients, on the topics and decisions discussed in consultations, is most successful in teaching new skills to professionals, but that the impact on overall health is contradictory, although limited data showed small positive effects on health status [12].

A cold, selfless doctor would have a nocebo effect on his patients. A doctor who shows empathy and cares about his patients fosters confidence, stresses expectations of regaining health and causes a strong placebo reaction [13].
Current research shows us that an adequate doctor-patient interaction sets in motion the same brain mechanisms as placebo, thereby assisting the medical treatment itself. Trust triggers a biochemical process. Participatory and non-tax communication fosters positive expectation about treatment outcomes. This type of communication influences $20-25 \%$ as a placebo effect [14].

There is a statistically significant effect between the type of health-patient relationship and clinical outcomes. The studies included compared the results between an intervention group, in which doctors, nurses and other health professionals received training in relational aspects such as eye contact, attention to the emotional or motivational interview strategies and a control group that provided standard attention [15].

To help our professionals achieve this type of skills we set out to work on this human factor project to make it a facilitator of this learning.

We start from the experience at Osakidetza in which there have been several projects that supported these claims.

The first of these experiences was the Project "Development of the Human Factor in the OSI Goierri - Alto Urola" carried out in the years 2012 - 2013 which was financed as a bottom up project by the Department of Health of the Basque Country and worked on the skills of active listening and participatory medicine. The project established a criterion of what is corrector incorrect in communication. This criterion allows a serious and technical foundation of communication styles and their effectiveness. We can say that the attendees were left with a clear idea of what good communication is [16].

Subsequently, the Human Factor Development Project was worked on in the Pediatrics Service of the Hospital of Cruces carried out in 2014 - 2015. This project was awarded by the Society of Medical Education of the Basque Country in 2016 as better communication under the title "Learning to humanize health care. Experience of the training project human factor in the Pediatrics Service of the University Hospital of Cruces" and was the promoter of an organizational and qualitative change of that service. Driven by the Department of Health and the management of the center, it has trained more than 300 service professionals. The main ob- 
jectives are: to foster an emotionally healthy and patient-centered relational culture, to improve human relationships in all the Pediatric Units of the Hospital Cruces, to train all those involved in direct care to provide tools that improve the interpersonal skills of professionals from a unified training. A common code encourage patient/family participation in their health care. Identify areas of improvement in processes that impact the treatment and actions for its approach [17].

Between 2015 - 2016 the Clinical Trial "Evaluation of the incorporation of the human factor into health outcomes of an care route on lumbalgia" was conducted. This was a multicenter, open and randomized clinical trial among 261 patients who attended 35 family doctors for episodes of acute lbp. Patients treated by doctors in the group who received specific training in communication skills and doctor-patient relationship responded to have better control of their symptoms, even if their visits to the doctor were minor (p-0.52).

But where a greater difference was observed was in the satisfaction of the doctors in the intervention group versus the control group. The former showed a higher degree of overall satisfaction in relation to the osteomuscular patient, as well as their sense of efficacy in the osteomuscular patient's approach was greater than the control group. This leads us to think that this specific training in human factor gives greater safety to the professional in the approach of this pathology.

Some research suggests that communication skills do not necessarily improve with experience, but with technique, so that is very important the training that trains in this type of skills. Based on this premise and the results of these Osakidetza projects we proposed to start this project of improving communication in our organization, adapting it to the characteristics of it.

\section{Objectives of the Study \\ Principal}

- $\quad$ Fostering a healthy, patient-centered relational culture

- Improving human relationships in units.

Specific

- Working and choosing the values of the Organization in a participatory way
- Encouraging patient/family participation in health care

- $\quad$ Training all people involved in direct care, providing them with tools that improve the interpersonal skills of professionals with a common code

- Identify areas of improvement in processes that impact the treatment and actions for its approach.

\section{Methodology}

The intervention is aimed at all the professionals of our Organization.

Three key elements considered as success factors for the project have been integrated into the human factor intervention design for OSI Tolosaldea. The first was the need to perform a previous work with the teams in which it will be implemented to generate a natural adherence to the project and create a culture favorable to change. The second was the identification of "change agents" who are recognized and supported by the teams. And the third was to define a plan of maintenance of the project that guarantees its continuity over time. All this, in addition, reinforced by leadership by professionals that will facilitate the progress of the project and its consolidation in the future.

To integrate all these elements, a triple intervention was proposed that impacted the elements cited, the most important was the leadership of the clinicians, another that we considered key was the generation of a culture in the organization favorable to change from the teams and to finish the own formative intervention that generates a new relational culture that is recognized as "style of patient care of the OSI Tolosaldea".

The key milestones of the project were six. The first was to develop an internal communication plan in the OSI for the presentation of the project and the awareness of professionals. To this end, a project management team was created in which both key OSI professionals and the reference hospital and even the social participation body in which the municipalities in the area participate could be integrated. It would be up to this project manager team to monitor and evaluate the project and facilitate its progress over time. The communication to the professionals was made in each UAP by its Unit Manager. In addition, a virtual communication space was created on a platform called Osakidetza's jakinsarea. 
Second, identifying change agents, a "change agent" is an organization professional who, in the change process initiated, will have the role of driving the project on your team. It may also be a reference, in the future, for the maintenance of the process over time.

Third, it was thought to facilitate the management of change from teams through value innovation. In this phase of the project it is about generating channels of relationship between the people of the different teams and between the different teams.

We understand that team cohesion is strengthened when the people who form them collaborate around a common goal that is accepted as a collective challenge.

In line with this purpose a challenge was proposed for the management of change in the teams. This challenge was intended to promote both cohesion and the involvement of professionals in the implementation of the project. It was focused on addressing decision-making based on shared values in the OSI and in each of the Primary Care Units. To this end, the teams worked with the definition of the values and their associated behaviors that would define the "OSI Tolosaldea care model".

Fourthly, there would be the own development of the Human Factor that is the cuore of the project.

Based on the previous experiences and the learning obtained, the following actions were proposed for the development of this part of the project:

- Previous fieldwork: Analysis of complaints, complaints and thanks to know the working context.

- $\quad$ Focus groups with patients to identify key elements of "treatment" by observational practice from the Design Thinking methodology.

- Development of evaluation questionnaires aimed at patients for the pre-launch phase of the project. Also aimed at professionals.

- Training of agents of change and professionals: Common code consisting of sought to prepare for the meeting, welcome the other correctly, ask to gather the information validating what it says and dialoguer to agree on the intervention together.
The content program was as follows

Session 1

Module 1: Human factor training project

- The Human Factor project at O.S.I. TOLOSA: Summary and results.

- How do we want to relate?

Module 2: The importance of human relations in the health field

- The importance of human relations in the health field.

- What do we mean when we talk about health treatment?

- The treatment and values in the care process.

- What is good treatment for patients?

Session 2

Module 3: The tool of the deal: communication

- How does communication work? From what is said to what is understood.

- The influence of stress and other emotional states on health-patient communication.

- Management of one's emotions: the key to treatment.

- How to regulate it?

Module 4: Good practice guide in 4 steps

- $\quad$ Paso 1: Prepare for the meeting

- Stop, connect and regulate one's emotions

- $\quad$ Reactive or proactive

- $\quad$ Listen-to-be so you can listen-to.

- $\quad$ Step 2: Establish relationship

- $\quad$ Connect with patient/partner

- $\quad$ Greeting, showing up (welcome)

- $\quad$ Eye contact, call him by name (recognize him).

- $\quad$ Step 3: Collect information

- Asking interested in the patient's perspective

- $\quad$ Listen 
- Validate your arguments and show emotional sensitivity.

- Step 4: Agree to the intervention together

- Dialogue, report, clarify and plan future sessions.

Planning of the intervention and follow-up period.

POST evaluation to patients and professionals.

And fifth, a future project maintenance plan was Plan de project maintenance designed, together with the different units and with the management team of OSI Tolosaldea. Sand agreed the strategy of reinforcement in the time of the project in the three areas that make up it: team reinforcement, leadership and human factor.

\section{Results}

Results of innovation by values

Group dynamics were carried out in June 2018 in which, in each UAP, the team of professionals identified the attributes that define them in their relationship with citizenship.

To this end, the facilitation sessions were asked to answer these four questions:

- What characterizes us as UAP?

- What things are important in our UAP?/What do we value?

- How do we think our patients/citizens see us?

- How do we want our patients/citizens to see us?

Each UAP made a diagram of the most commonly used expressions and terms in the responses given by the people on the teams and was proposed to work on identifying the behaviors associated with each of them.

The values most frequently repeated in all UAPs were accessibility, resolution, closeness, collaboration, respect, communication and professionalism.

Within the framework of the Human Factor Project initiated in the OSI Tolosaldea during the months of February and March 2019 facilitation sessions were held in the five UAPs with the aim of having the professionals of these teams collaboratively define the val- ues and behaviors that are key in health care to the public and in the relationship between the people of the team and that identify the behaviors associated with these values.

For the definition of the behaviors associated with each of these values, the facilitation sessions asked the people on the teams to relate at least two day-to-day behaviors based on real-world situations. They were in a time to do so using phrases initiated with an infinitive action verb that would define behavior. Sessions reinforced the need to identify observable behaviors that could be measurable, avoiding general references. They were also asked to identify behaviors in relation to patients and in the relationship with the people on the team in a differentiated manner.

With the contributions of all the people in the team, the last part of the session was dedicated to, in pairs or threesomes, making a summary proposal. This summary has worked to make the final proposal contained in this report.

We encounter two difficulties in dealing with the work of synthesis. One was that, although a total of thirteen different expressions had been worked on in each UAP's teams, however, the identified behaviors have been repeated in several of them; which might indicate that they are not distinct entities despite being referred to differently. And the other that in the identification of behaviors there is a tendency to use phrases of a general nature with terms not in all cases associative to specific behaviors.

From the analysis of the total contributions received, it was noted that professionals from the five teams of the OSI Tolosalde highlighted behaviors related to the following aspects involved in the relationship with patients and colleagues. Para these professionals, are inherent in the provision of a public health service in the OSI Tolosaldea and, therefore, definers of their style of care and relationship. And these behaviors are professionalism, current knowledge hoisted and shared, collaboration in the team, respect for the people of the team, effective communication with the patient and human treatment in the relationship with patients from the empowerment.

Definitions of each were proposed on the basis of the inputs contained in the analysis. 


\section{Professionalism}

OSI professionals Tolosaldea understand professionalism as a commitment to citizenship, organization and equipment.

Commitment to citizenship that materializes in giving an adequate health response to the population through personalized attention, based on scientific evidence and updated knowledge, guaranteeing confidentiality in their action and the privacy of people.

Commitment to the organization that materializes in the active contribution in achieving the objectives of the UAP and in the proper use of available resources.

Commitment to the team that is specific to active participation in team sessions and in the willingness to agree on common criteria of action among the people of the team.

Professionality is embodied in the following behaviors:

- $\quad$ To care for patients who come to our centers by offering them the best solution without delay, taking to another level what we cannot solve.

- $\quad$ Conduct personalized care to our patients based on clinical and care decisions on scientific evidence according to established protocols.

- $\quad$ Adjust appointments to promote family and work reconciliation of patients.

- Know the objectives of the unit and actively contribute to its achievement.

- $\quad$ Actively participate in team sessions, promoting consensus in decision-making and the adoption of common criteria for action.

\section{Sharing knowledge}

OSI professionals Tolosaldea stressed the need to keep scientific and professional knowledge up-to-date as a key element in providing a quality public health service. Sharing knowledge among professionals is a practice they considered essential for the care of the population, which encourages collaboration between the people of the team and facilitates a better response to patients.
The Current and shared knowledge, as the value of a primary care team is concreted in the following behaviors:

- $\quad$ Share clinical cases of interest in team sessions.

- Accompany resident staff in learning.

- Inform the team of the news that is taking place in the professional, scientific, organizational, etc.

\section{Collaboration in the team}

OSI tolosaldea professionals understood collaboration as the willingness of the people of the team to collaborate when required by providing their skills and knowledge. It involves an attitude of helping the partner from respect for their contributions, avoiding judgments and accepting different points of view and different ways of working. Working as a team at OSI Tolosaldea involves fulfilling the role of each and seeking coordinated attention to users among different professionals.

Team collaboration is embodied in the following behaviors:

- Treat patients in a partner if I do not have patients at that time.

- Answer questions and questions from teammates about the processes in which I take part.

- Accept the team's decisions as their own.

\section{Respect for people on the team}

OSI professionals Tolosaldea considered respect for the people of the team as an attitude by which all UAP members recognize the same, regardless of their professional status or position in the organization. Respect implies the ability to accept different points of view and different ways of acting in the face of everyday situations. Respect among people on the team goes beyond strict observance of basic standards of education; it involves fostering close, empathetic and trust-based relationships.

The respect to the people of the team is concrete in the following behaviors:

- Do not interrupt the use of peers in professional meetings or conversations.

- $\quad$ Arrive on time to meetings, work appointments, consultation, etc. 
- $\quad$ Target the people on the team in a calm way and without raising your voice.

- $\quad$ Avoid value judgments in public about peer work.

- $\quad$ Take an interest in the personal well-being of team members.

\section{Effective patient communication}

OSI Tolosaldea professionals considered communication with patient one of the main success factors in the therapeutic relationship with patients. Effective communication strengthens the link between health professionals and patients by fostering mutual trust. The keys to patient communication in OSI Tolosaldea are based on giving importance to the reception, on the use of adequate and understandable language and on the constant inquiry of patient needs. All this in a friendly, accessible and mutually respected environment.

Effective communication with patient is embodied in the following behaviors:

- Welcome the patient with a smile and a kind gesture when he or she goes to the consultation or the AACs.

- Use understandable language, making sure you understand what we're saying. If it helps the purpose of communication we will use images to accompany the information.

- $\quad$ Constantly investigate and validate the information provided to us by the patient.

- Communicate with patients in the language of your choice.

- Give objective information without judgment.

Human treatment in patient relationship from empowerment OSI professionals Tolosaldea understand the relationship with patients from the acceptance of the wealth provided by the religious, cultural, ideological, sexual or other diversity of the people who come to the health centers. Closeness in treatment, empathy with especially vulnerable people or physical restorative contact, contribute to strengthening a human environment compatible with new technologies in the service of health. All this based on a relationship of peers that respects the decisions of the patient.
The human treatment in the relationship with patients is concreted in the following behaviors:

- $\quad$ Treat patients with love.

- Show closeness through restorative physical contact, especially in the most delicate moments.

- Focus attention on the patient by looking them in the eye, avoiding focusing on the computer.

- $\quad$ Protect patient privacy.

- Do not answer the personal phone in consultation in front of patients.

- Accept the decisions patients make about their health, even if they don't share them.

Results of patient focal groups

Two patient focus groups were performed at two Health Centers.

One of the main conclusions from the analysis of the information collected is that patients consider that the "good treatment" is to be treated as people. Through the good treatment of yours and yours, you look at them, listen to them, consider your opinion and be cordial. For patients it is important that your referring doctor is always the same, since not knowing the professional who cares for them generates distrust. Waiting times are also very important, both to get an appointment and to enter consultation, and this is considered by patients as an upgradeable aspect. They also consider the time spent for the consultation to be insufficient.

Patients noted the importance of information and how it is transmitted, as it is sometimes not understandable because it is overly technical. They also believed that closeness and accessibility was a factor they considered important.

In small populations where everyone is known, contact is closer and professionals more accessible.

\section{Training evaluation results}

The intervention programme consisted of the implementation of 9 training groups, one from the Gestor Group with seven hours of 
training. And eight other groups of healthcare professionals with 6 hours of training each.

After the training sessions, attendees completed a questionnaire to evaluate the training carried out.

In this evaluation, the values that refer to "the interest of the contents" with a 7.8, "the relationship with my colleagues" and "the adequacy of the room and the furniture" with a 7.7 stand out with higher scores. The lineup had an overall score of 7.5, out of 10 .

\section{Results of pre and post patient surveys}

The impact assessment was carried out through the "Relationship and Treatment" questionnaire consisting of 11 items sensitive to the skills trained in the intervention. Study the change in the perception of treatment received by patients at OSI Health Centers Tolosaldea before and after the intervention. It was conducted through a questionnaire (Ad Hoc) on the relationship and treatment between health-patient to a sample of 82 patients in the preintervention and 176 in the post-intervention.

First, an analysis of the PRE sample was carried out, collected before the intervention, in which it was observed that patients considered that the treatment received from professionals was very good 8.7 (out of 10). This statement was also corroborated during the development of the discussion groups, in which patients who participated in the two groups expressed satisfaction with the treatment received in health facilities, and the progress that has been made in recent years in this regard.

Subsequently, patients from the OSI Health Centres Tolosaldea were asked to complete the POST "Relationship and Treatment" questionnaire. No significant differences were observed as we started from very good initial results. $66 \%$ of patients in the PRE questionnaire believe that the treatment received by professionals is between 9 and 10,on a scale of 1 to 10 , being 1 very bad and 10 excellent.

\section{Perception of change questionnaire results}

After the intervention, the 138 trained professionals were asked to complete the Project Change Perception questionnaire.
A total of 66 questionnaires have been collected, the analysis of which is described below:

The question of the extent to which do you consider that the $\mathrm{Hu}$ man Factor Development Project has contributed to improving the relationships/communication between professionals and patients in the OSI Tolosaldea? has obtained an average of 5.1 (out of 10), with a fashion of 7.

Professionals have given an average of 5.1 (out of 10), to the question to what extent you consider that the Human Factor Development Project has contributed to improving relationships/ communication between teammates at the centre. With regard to the question, to what extent do you consider that the Human Factor Development Project has contributed to establishing a common communication code shared by professionals in OSI Primary Care Units Tolosaldea? has obtained an average of 4.7 (out of 10).

Professionals have pointed out as positive aspects of the project:

- The direction of the project. The transmission format. The focus. It allows the members of the working group to reflect and, after valuing, improves relationships with colleagues and patients.

- $\quad$ Being aware of the diversity of people in relationships facilitates communication. Learning techniques to improve communication is the key to positive results.

- It has helped improve the environment in one of our centers. Professionals have seen it as a way of helping to improve communication between them.

- Project that has helped to work the management of emotions.

- The training provided has been very good.

- Discuss communication and relationship issues between people that we don't usually deal with. There is little training about it.

- $\quad$ During the training we talked about many interesting topics and situations and in my case has helped me in the relationship with some patients and situations.

- It gets on the table, almost without realizing the good and the bad of the team. 
- In times of conflict with people (patients and peers) I think before acting.

- It reminded us that we worked with people and not machines.

- $\quad$ Accept the new reality of UAP.

- It's hard to change something we've been doing all our lives.

- It serves to think of things we didn't usually stop to think about.

- Interrelationship between professionals from different stages.

- $\quad$ Be aware of my abilities to manage to control my mood, reactions to provocations, anxiety.

- What you've learned on a personal level.

- Talk about topics that had never been discussed at our Health Center. Propose improvements.

\section{Discussion}

- In this type of project it is very important to have a tractor equipment that exercises leadership to drive the achievement of it.

- Working values from the perspective of professionals acts as a facilitator to feel the culture of the organization as their own.

- Participants can improve their personal relationship as this intervention encourages sharing of beliefs and values in a relaxed environment for this.

- The second phase, the work on innovation in values, is oriented towards the creation of a favorable environment for further training in the next phase. This helps direct her toward changing patient-centered orientation, making them part of decisions regarding her health.

- This unified training has provided healthcare professionals with tools that improve interpersonal skills for a better doctor-patient relationship and also between professionals. It has given them a common code when it comes to relating to patients and they have been able to learn to listen more to patients. And with all this, the relationship with the patient/family has been improved in the development of health care skills.
Conclusion

- The results of the patient group discourse analysis and the analysis of the PRE and POST questionnaires show that patients consider the treatment received by professionals very good. The project was already based on very high satisfaction values.

- No differences are observed in the descriptive analysis of PREPOST questionnaires to patients. This could be explained by the difference between the number of pre- and post,82 and 176 questionnaires respectively which makes it very difficult to extrapolate any conclusion. The lengthening in the time of the post measurement, almost 3 months, while the PRE was done in two weeks may partly explain the behavior of the data and its apparent contradiction with qualitative comments.

- There is a very significant difference in how they evaluate the project quantitatively and qualitative comments: Healthcare professionals value with fairly low average scores (4.7 - 5.1) the issues of the impact of the Human Factor Project, however, qualitative assessments refer to having noticed an improvement in the relationship with their peers after the intervention, as well as having used the communication tools, developed during training.

- We emphasize that professionals demonstrate as very positive the being able to share values in the way of understanding human relationships and that the project has allowed to generate discussion and reflection about how to communicate.

- The results obtained so far allow to affirm that the intervention of Human Factor causes favorable changes in the indicators of improvement of the treatment perceived by patients.

- Significant changes in specific skills and behaviors have been detected: professionals are more present, aware of particular needs, show more interest, and ask patients more. In addition, patients have felt more listened to.

\section{Bibliography}

1. M Simpson., et al. "Medical-patient communication: the Toronto Consensus Report”. BMJ (1993). 
2. DL Roter., et al. "Physician-patient communication: the relationship with malpractice claims among primary care physicians and surgeons". JAMA 277 (1997): 553-559.

3. Stewart MA. "Effective physician-patient communication and health outcomes: a review". Canadian Medical Association, Journal 152 (1995): 1423-1433.

4. Communicating with Patients. Advice for medical practitioners. Australian Government. National Health and Medical Research Council (2004).

5. Paul Little., et al. "Observational study of effect of patient centredness and positive approach on outcomes of general practice consultations". BMJ 323 (2001): 908-911.

6. Jairo N Fuertes., et al. "The physician-patient working alliance". Patient Education and Counseling 66 (2007): 29-36.

7. Kaplan SH., et al. "Assessing the effects of physician-patient interactions on the outcomes of chronic disease". Medical Care 27 (1989): S110- S127.

8. Griffin SJ., et al. "Effect on health-related outcomes of interventions to alter the interaction between patients and practitioners: a systematic review of trials". Annals of Family Medicine 2.6 (2004): 595-608.

9. M Muñoz., et al. "Evaluation of a patient-centred approach in generalized musculoskeletal chronic pain/fibromyalgia patients in primary care". Patient Education and Counseling 48 (2002): 23-31.

10. C Hoffmann., et al. "Patients' expectations of acute low back pain management: implications for evidence uptake". BMC Family Practice 14 (2013): 7.

11. J Verbeek., et al. "Patient Expectations of Treatment for Back Pain. A Systematic Review of Qualitative and Quantitative Studies". Spine 29 (2004): 2309-2318.

12. "Interventions for the promotion of a patient-centered approach by professionals in clinical consultations (Translated Cochrane Review)". Cochrane Database of Systematic Reviews (2012).
13. Oishansky B. "Placebo and nocebo in cardiovascular Elath: implications for healthcare, research and doctor patient Relationship". Journal of the American College of Cardiology (2007): 415-421.

14. Tor D Wager., et al. "Placebo induced changes in the anticipation and experience of pain". Science (2004): 1162-1167.

15. Kelley JM., et al. "The Influence of the Patient-Clinician Relationship on Healthcare Outcomes: A Systematic Review and Meta-Analysis of Randomized Controlled Trials". PIoS ONE 9.4 (2014): 94207.

16. Armentia Macazaga JM and Cancio H. "People and the human factor: keys to cultural change and improvement in integrated health systems". RISAI 7.1 (2015): 1-11.

17. Astigarraga Aguirre I. "Many essential people to face the challenges of child and adolescent care". Spanish Journal of Pediatrics 71 (2015): 190-191.

\section{Assets from publication with us}

- Prompt Acknowledgement after receiving the article

- Thorough Double blinded peer review

- Rapid Publication

- Issue of Publication Certificate

- High visibility of your Published work

Website: www.actascientific.com/

Submit Article: www.actascientific.com/submission.php

Email us: editor@actascientific.com

Contact us: +919182824667 